\title{
Self-tuning transmitter for fibre-to-the-antenna PON networks
}

\author{
P. Parolari a,*, L. Marazzi ${ }^{\text {a }}$, M. Brunero ${ }^{\text {a }}$, A. Gatto ${ }^{\text {a }}$, M. Martinelli ${ }^{\text {a }}$, P. Chanclou ${ }^{\text {b }}$, \\ Q.Deniel $^{\text {b }}$, F. Saliou ${ }^{\text {b }}$, Sy Dat Le ${ }^{b}$, R. Brenot ${ }^{c}$, S. Barbet ${ }^{c}$, F. Lelarge ${ }^{c}$, \\ S. Gebrewold ${ }^{\mathrm{d}}$, S. O'Duill ${ }^{\mathrm{e}, 1}$, D. Hillerkuss ${ }^{\mathrm{d}}$, J. Leuthold ${ }^{\mathrm{d}}$, G. Gavioli $^{\mathrm{f}}$, P. Galli ${ }^{\mathrm{f}}$ \\ a Politecnico di Milano, Dipartimento Elettronica Informazione e Bioingegneria - PoliCom, via Ponzio 34/5, 20133 Milan, Italy \\ ${ }^{\mathrm{b}}$ Orange Labs, 2 Avenue Pierre Marzin, 22307 Lannion, France \\ c III-V Lab, Route de Nozay, 91460 Marcoussis, France \\ d Swiss Federal Institute of Technology (ETH), 8092 Zürich, Switzerland \\ e Karlsruhe Institute of Technology (KIT), Germany \\ ${ }^{\mathrm{f}}$ Alcatel-Lucent Italia, Via Trento, 3020871 Vimercate, Milano, Italy
}

Available online 24 January 2014

\section{Introduction}

Fibre-to-the-home (FTTH) access for residential market will be deployed through Passive Optical Networks (PONs) essentially based on time division multiple access. Conversely, pure Wavelength Division Multiplexing (WDM) PON so far has not been considered as a solution for this residential market. On the other hand, WDM PON offers a pair of wavelengths per user and achieves point-to-point connectivity, this allows to share the medium while supporting independence of multiple access protocols.
As the current provided capacity is $1 \mathrm{Gbit} / \mathrm{s}$ and $10 \mathrm{Gbit} / \mathrm{s}$ is foreseeable, WDM PONs are good candidates for mobile applications.

In this manuscript, we focus on digital radio over fibre (D-RoF), where, thanks to software defined radio (SDR), the analogue processing is performed in the remote radio head (RRH) and is located near the antenna, while the baseband digital processing is separately performed in the base band unit (BBU). The BBUs can thus be centralised in a data centre or base station hotel similarly to a cloud computing model, the cloud radio access network ( $\mathrm{C}$ RAN), and can be connected to the corresponding RRH by means of optical fibres. The C-RAN creates new fibre network requirements to achieve the fibre-to-the-antenna (FTTA), which, differently from the fibre-wireless (FiWi) access usually exploiting an optical distribution networks

\footnotetext{
* Corresponding author. Tel.: +39 0223998926.

E-mail addresses: paola.parolari@polimi.it,

p_parolari@yahoo.it (P. Parolari).

${ }^{1}$ Now at: The Rince Institute Dublin City University, Dublin, Ireland.
} 
based on power splitters, could be supported by other fibre-network solutions. In particular the link between BBU and RRH can also be called "fronthaul" and transports the aggregated traffic and the mobile-network synchronization signal. The Common Public Radio Interface (CPRI) is one of the standards for the fronthaul interface, it has stringent low-latency requirements, symmetrical bit rates ranging from 0.6 to $10 \mathrm{Gbit} / \mathrm{s}$ for up- and downstream, and strong require-ment on jitter.

Actually the simplest optical distribution network between RRH and BBU can be achieved by point to point fibres: each RRH (for example corresponding to a sector) is connected directly to the BBU using one or two fibres. This solution is expensive in terms of number of fibres per antenna site due to the high RRH number per antenna site. Therefore, a multiplexing technique is needed to transport several RRH-BBU links. The OTN networks are a mature technology to achieve a multiplexing function but require power supplied equipments (i.e., active equipments), not very likely to be placed on antenna sites, and specific implementation to achieve the CPRI requirements with the needed frequency accuracy [1-3]. Standard PON technology faces limits both for maximum bit rates and for TDMA ranging mechanisms, which induce jitter issues for other traffic [4]. The WDM PON solution overcomes these limitations. Although coarse WDM transceivers could be a first pragmatic approach, in case of massive deployment, inventory problems could burden the mobile network administration, thus colourless transceivers are to be preferred as they will limit inventory and maintenance costs [5-7]. Colourless transceivers employing a wavelength tuneable optical source have the main drawback of the wavelength assignment policy: the transceiver has to host all the functions to discover, assign, and maintain the adequate wavelength on their boards [8].

Thus preferred WDM PON solutions should be both colourless and self-tuning, i.e. able to automatically and passively assign the wavelength source. Externally-seeded solutions for re-modulation suffer scattering impairments, due to both the Rayleigh and the Brillouin backscatterings, which strongly limit the distance between the central office and the ONU [9]. WDM transceivers with spectrally sliced broadband optical sources have a limited optical budget and bit-rate reach due to inherent excess intensity noise and chromatic dispersion [10]. Transceivers based on self-seeded optical sources, as we are proposing, are both colourless and self-tuning. Moreover, avoiding external sources, they do not suffer from any backscattering. Finally the presence of an external cavity in principle should allow a promising performance both in terms of power budget and of ultimate bit rate [11].

In this contribution we thus focus on the proposal of a network embedded self-tuning colourless transmitter, based on reflective semiconductor optical amplifier (RSOA) selfseeding architectures [11]. The next sections will present the C-RAN requirements and possible network solutions; in particular the preferred solution based on the network embedded self-tuning colourless transmitter is discussed by showing its principle of operation, the development and the modelling of the active elements suitable for sustaining the self-tuning transmitter and finally by presenting recent experimental results.

\section{Context of cloud radio access network and fronthaul requirements}

C-RAN is a new RAN architecture concept that may change the existing paradigm and the way the RAN evolves in future. Future generations of macro base stations will rely on distributed RRHs, which contain the RF transmitter and receiver components (including power amplifier, duplexer, low noise amplifier etc.) and which can be mounted directly on the antenna mast. Due to their lower capital and operating expenditures, these RRHs are currently being deployed both for new technologies (e.g. LTE, WiMax, and MIMO) and for older technologies (2 G, $2.5 \mathrm{G}$, and $3 \mathrm{G}$ ) replacement infrastructures. The RRH can be linked to the BBU by a single mode optical fibre using a digital radio signal $[12,13]$ (D-RoF) with a standard interface for the baseband signals such as CPRI [14] or Open Base station Architecture Initiative (OBSAI) [15]. This architecture allows the possibility to move the BBUs to a central office (CO) (Fig. 1) obtaining BBU centralisation or BBU hostelling with stacking: BBUs of different basestations are co-located in the same CO. Generally there is one BBU per Node B site and they communicate with other BBUs within the BBU hostel via standardized $\mathrm{X} 2$ interface.

A further improvement is provided by the C-RAN or BBU hostelling with resource pooling between all the BBUs in the BBU hostel [16] (Fig. 2). A centralised set of BBUs with resource pooling can handle a large number of RRHs located at different antenna sites. Both architectures, due to the baseband unit breakdown, need a new connectivity segment, called fronthaul, between the RRHs on the cell site and the BBUs in the CO.

The fronthaul characteristics depend on the cell site technologies and dimensions; bandwidth requirements between the cell site and the $\mathrm{CO}$ where the BBU is localised will differ from case to case. CPRI and OBSAI transport standards share similar features, here in particular we focus on four important characteristics of CPRI, that is bit rate, latency, jitter and synchronization, and fibre architectures.

\subsection{High bit rate capacity needs of fronthaul}

Based on radio technologies the CPRI link rates can range from $614.4 \mathrm{Mbit} / \mathrm{s}$ to $9.8 \mathrm{Gbit} / \mathrm{s}$. Table 1 gives the typical CPRI data rates corresponding to 1 carrier and 1

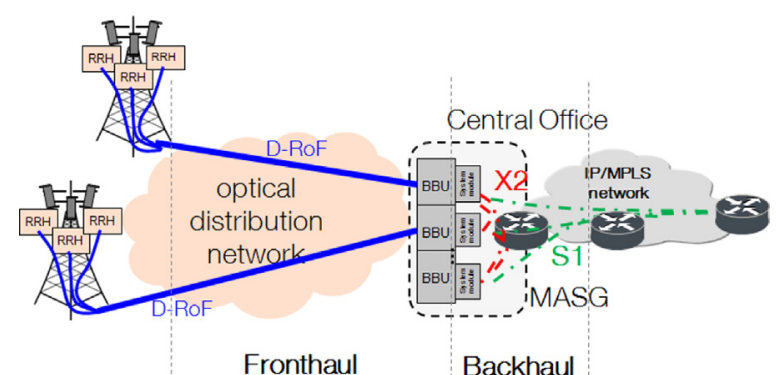

Fig. 1. BBU hostelling with stacking based digital-radio over fibre link over optical distribution network, the BBUs are collocated in a $\mathrm{CO}$ and are connected to the radio towers through an optical distribution network. 
sector for different radio technologies. The calculation of data rate per CPRI link is based on the following expression:

Data rate $=M \times \mathrm{Sr} \times N \times 2(I / Q) \times 10 / 8$

where $M$ is the number of antennas per sector (as in multiple-input and multiple-output), $\mathrm{Sr}$ corresponds to the sampling rate (sample/s/carrier), $N$ is the sample width (bits/ sample), $2(I / Q)$ is the multiplicative factor accounting for inphase $(I)$ and quadrature-phase $(Q)$ data, and the 10/8 factor takes account of the $8 \mathrm{~B} / 10 \mathrm{~B}$ coding.

The requirements for the new fronthaul connectivity segment between RRH and BBU, are strongly related to CPRI data requirements. The logical link between antenna sites and BBUs has to consider several CPRI links at different bit rates in relation with symbol rate, sampling rate, sampling time, carrier number and antenna number, whereas it is independent of the modulation scheme.

\subsection{Latency constraints on the fronthaul}

Since the link between RRH and BBU is at the physical radio signal level, the total latency that the radio signal can tolerate has to include the fronthaul latency. The most critical constraint comes from the up-link synchronization method, the Hybrid Automatic Repeat Request (HARQ). In case of retransmission, this parameter has a direct impact on the peak data per user. The fronthaul latency can be calculated subtracting the mobile equipment processing time: considering the maximum timing advance $(667 \mu \mathrm{s}$, for LTE) and a $10-\mathrm{km}$ cell radius, the round trip time available for propagation between RRH and BBU is only $700 \mu$ s for LTE and $400 \mu \mathrm{s}$ or less for LTE-Advanced (including Coordinated MultiPoint (CoMP)). The round trip time includes both fibre-propagation time delays and link equipment processing. BBU and RRH are preferably connected without active equipments, thus in this case, LTE-Advanced time delay allows for maximum $40-\mathrm{km}$

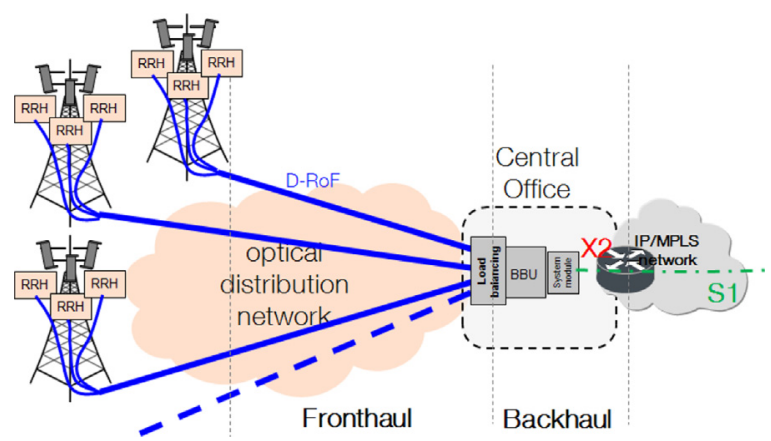

Fig. 2. BBU hostelling with resource pooling (C-RAN), a single baseband unit is connected to a number of radio towers to increase the overall network bandwidth through resource pooling. reach between $B B U$ and $\mathrm{RRH}(80 \mathrm{~km}$ round trip time with propagation time in $20 \mathrm{~km} \approx 100 \mu \mathrm{s}$ ).

\subsection{Jitter and synchronization}

The CPRI specifications for synchronization are included in the CPRI-frame final part and they define the maximum jitter transfer bandwidth of the employed Phase-Locked Loop (PLL). For example the LTE base station air interface frequency accuracy is $\pm 50 \mathrm{ppb}$ (parts per billion). This requirement sets a constraint on the fronthaul frequency synchronization, which is expressed as a contribution to the overall requirement (e.g. a suitable portion of $50 \mathrm{ppb}$ ). Typically a budget of $2 \mathrm{ppb}$ is allocated to the BBU-RRH link. Some options of LTEAdvanced require phase synchronization, thus a phase accuracy requirement budget is allocated to the CPRI link and the contribution of the link itself shall be taken into account. It is appropriate that both phase noise and asymmetries are considered (in fact the error in the delay measurement is also impacted by asymmetry in the CPRI link).

\subsection{Fibre resources availability for fronthaul}

Optical fibre or wireless transmission technologies are needed at the cell site to connect BBU and RRH. For some simple radio configurations microwave links could be an option. Nevertheless due to its large bandwidth the fibre is the preferred option for LTE traditional backhaul and it is the standard solution to connect the fronthaul. Three main topologies can be foreseen for the optical distribution network between RRH and BBU.

- Point-to-point: each RRH (for example corresponding to a sector) is connected directly to the BBU. This solution could be expensive as the number of fibres per antenna site grows quickly. Therefore, wavelength multiplexing of CPRI channels would be desirable to achieve virtual point to point connectivity.

- Daisy chain: several RRHs could be cascaded (with time division multiplexing of each RRH data rate) towards the BBU. This topology allows for a reduction of the number of fibres but at the same time introduces a single point of failure.

- Multi-path: ring and mesh topologies have the advantage of addressing the issue of network availability by closing the chain and providing an alternative path to maintain connectivity between the BBU and the RRHs in the presence of a link failure on any of the segments in the ring.

The fronthaul network segment thus needs an optical fibre infrastructure and equipments with versatile bit rate, a

Table 1

Typical data rates of CPRI in function of radio technologies.

\begin{tabular}{|c|c|c|c|c|c|c|c|c|}
\hline RAN & GSM 1T1R & GSM 1T2R & WCDMA 1T1R & WCDMA 1T2R & LTE $10 \mathrm{MHz} 2 \times 2$ & LTE $10 \mathrm{MHz} 4 \times 2$ & LTE $20 \mathrm{MHz} 2 \times 2$ & LTE $20 \mathrm{MHz} 4 \times 2$ \\
\hline CPRI & 12.304 & 24.608 & 307.2 & 614.4 & 1228.8 & 2457.6 & 2457.6 & 4915.2 \\
\hline Data rate & Mbit/s & Mbit/s & Mbit/s & Mbit/s & Mbit/s & Mbit/s & Mbit/s & Mbit/s \\
\hline
\end{tabular}


reach of up to $20 \mathrm{~km}$, without significant impact on jitter and synchronization and possibly offering demarcation points [17] and CPRI multiplexing and a passive and compact form of transceiver at the antenna site is also desirable to avoid power consumption.

Among existing optical solutions to achieve the CPRI data transport which satisfy the above constraints we focus on a point-to-point WDM PON solution (Fig. 3) based on RSOA self-seeding architectures as it offers a passive solution at the antenna site with WDM transceiver compatible with standard Small Form-factor Pluggable (SFP). Moreover this solution is based on colourless and selftuning transceivers allowing to reduce inventory issues and to have an automatic and passive mechanism for the wavelength assignment, based on the connection with a port of the optical multiplexer (MUX). In the next sections we will describe in detail the architecture and the principle of operation of the transceiver based on the RSOA selfseeding.

\section{Network embedded self-tuning colourless transmitter principle of operation}

The access network optical network unit (ONU), which will be hosted on the antenna site, houses a transmitter physically coinciding with a portion of the network itself. The transmitter relies on a laser cavity, which comprises an RSOA active chip, placed at the ONU, whose reflective end is one of the cavity mirrors, the distribution fibre, which connects the ONU and the remote node (RN), the $\mathrm{RN}$ array wave-guide (AWG) multiplexer and a mirror also placed at the RN. The AWG represents the optical multiplexer and is the cavity wavelength-selective element.

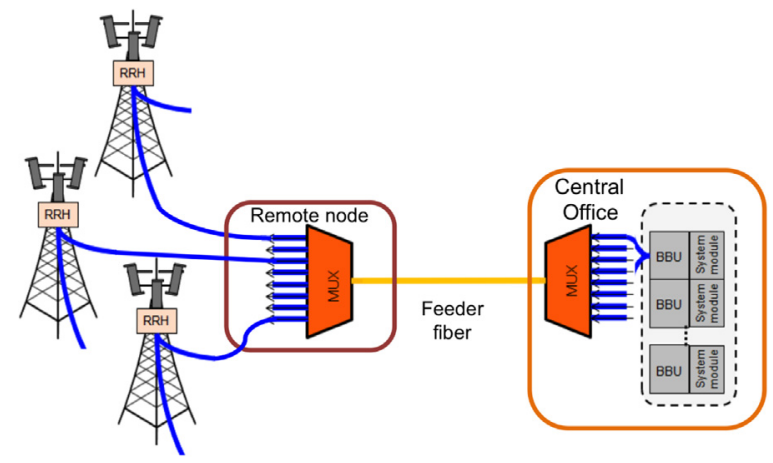

Fig. 3. Proposed point-to-point WDM PON solution for the fronthaul.
Each cavity associated to the single AWG channel will emit at the channel wavelength, self-tuning itself and thus achieving a colourless transmitter, allowing for an automatic and passive assignment of the wavelengths. The RSOA is the cavity active element and it sustains the cavity lasing, overcoming the overall cavity losses. The RSOA is also the modulating element, through direct modulation of the active chip current. Finally the RSOA allows recirculating modulation cancellation through the self-gain modulation mechanism, supporting new signal modulation.

A major issue with RSOA self-seeded architecture is the polarisation evolution within the optical circuit [18]. The problem has been solved for low polarisation dependent gain (PDG) RSOA exploiting the properties of the Faraday rotator mirror (FRM) [18,19].

To allow the employment of high performance RSOAs, presenting high PDG, the addition of a Faraday rotator (FR), located in close proximity to the RSOA output, and a FRM, shared by all ONUs and placed at the RN close to the AWG (Fig. 4) is necessary. Thanks to the property of the FR it has been demonstrated [20] that at the RSOA input the state of polarisation is stably retraced and aligned with the RSOA high-gain transverse mode, showing a high degree of polarisation.

The principle of operation of the self-tuning transmitter clearly evidences the necessity to study the characteristics of the required gain device and possibly to develop new RSOAs as motivated by the peculiar triple role sustained by this key component. This detailed analysis is presented in the next two sections.

\section{Active devices for network-embedded self-tuning transmitters}

The active devices able to support the proposed transmitter should necessarily provide a very large gain. The gain typically should exceed $20 \mathrm{~dB}$ even when in deep saturation in order to exceed the overall cavity losses. Moreover RSOA devices for future-proof self-seeded transmitters should allow for high-speed direct modulation. Fig. 5(a) shows the eye diagrams obtained with RSOA current direct modulation at different bit rates and for various RSOAs having similar gain values (25-30 dB), made either with quantum dashes (QD), or with multiquantum wells (MQW), or with bulk material having an optical confinement of $20 \%$ or $80 \%$. As can be seen by comparing the eye diagrams the MQW device is the fastest

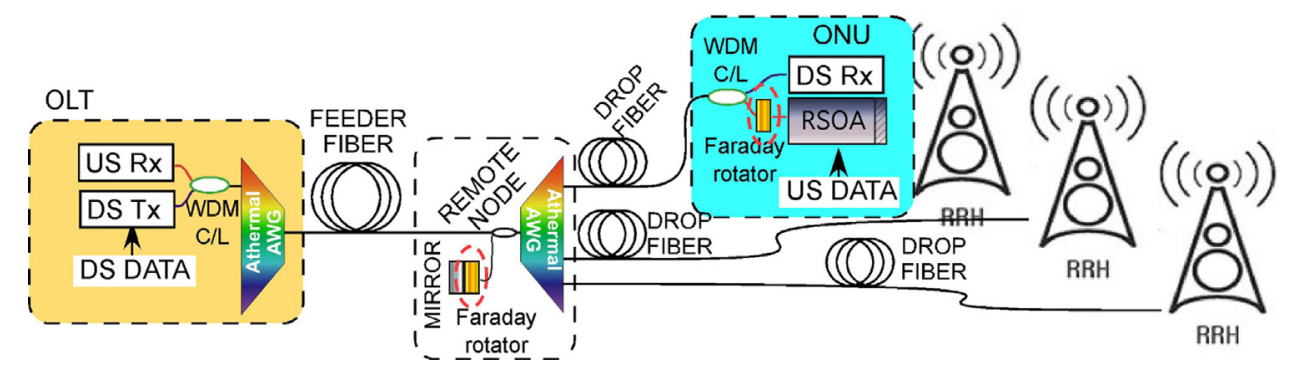

Fig. 4. Two-FR based self-tuning architecture. 

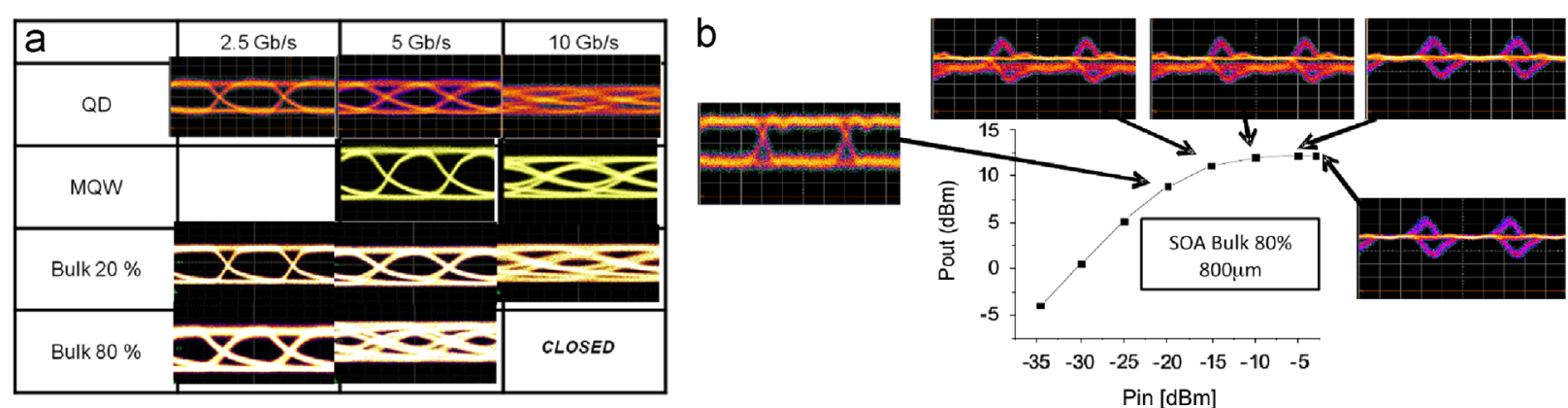

Fig. 5. (a) Eye diagrams of directly modulated RSOAs operated at 2.5, 5 and 10 Gbit/s (pure ASE modulation) for QD, MQW, and bulk (with an optical confinement of $20 \%$ or $80 \%$ ) RSOA. (b) Signal cancelation effect in RSOA as obtained by the nonlinear transfer function. The eye diagrams are taken for $5 \mathrm{Gbit} / \mathrm{s}$ signals with a bulk RSOA that has an optical confinement of $80 \%$.

together with $20 \%$ bulk one. Further the ability to cancel the recirculating signal, which is based on gain compression, has to be considered. Fig. 5(b) shows the static and dynamic transfer function of a bulk RSOA with an optical confinement of $80 \%$. The input signal presents an extinction ratio of $5 \mathrm{~dB}$ and data cancellation is fast and efficient for an input power above $-10 \mathrm{dBm}$.

To operate at high bit rates and with low input powers, the gain should be as large as possible, and the optical/ optical $(\mathrm{O} / \mathrm{O})$ modulation speed should be very large as well. These requirements can be achieved using very large optical confinements, which improve the efficiency and the speed of data cancellation. Data cancellation with MQW RSOA is inherently less efficient because of a lower optical confinement. Nevertheless the lack of confinement can be partially compensated by using long devices. Thus the ideal candidate for self-tuning transmitters should be MQW RSOAs for high speed data modulation and large confinement bulk RSOAs for recirculating data cancellation; a $1 \mathrm{~mm}$ long MQW RSOA would be a good single device compromise.

\section{Models for the network-embedded self-tuning transmitter}

Central to the self-seeded network embedded transmitter is the RSOA device, whose model is then incorporated into a larger model for the network embedded transmitter. The RSOA provides three important functions: amplification, data cancelation and direct modulation. The developed model is based on implementations of travelling wave amplifiers and the mathematical model includes many device parameters, which can be found in [21]. The model was at first compared with experimental results with CW and externally modulated seeding conditions, showing excellent agreement between the calculated and measured results in static conditions [21]. Moreover the comparison of simulated eye diagrams with experimental results in different saturation regimes confirmed the adequacy of the model to predict the dynamic behaviour of RSOAs [21]. The modelling of the RSOA operating in a self-seeded cavity is presented in the schematic of Fig. 6 . A laser field builds up due to recirculating ASE, and then the intensity of the outgoing wave is modulated via the RSOA injection current; the recirculating modulation returning to the $\mathrm{RSOA}$, is cancelled as it propagates through the RSOA thanks to the decoupling of the gain modulation with gain saturation. Satisfactory operation of a self-seeding source is only obtained if the cancelation condition is met. Simulations at first determined the current injection allowing to cancel the modulation component of the recirculating signal, by matching cavity losses and the location of maximum output power in RSOA gain versus output power plots. The simulation platform is created by fixing a time delay of $400 \mathrm{~ns}$ ( 1000 bits at $2.5 \mathrm{Gbit} / \mathrm{s}$ ) between the time when the outgoing US signal leaves the RSOA and when the signal returns as a recirculating signal to the RSOA. The laser field is built up from ASE and once a steady state field is established then the current is modulated. The simulated laser output power versus current $(L-I)$ curve displays the typical threshold condition for lasers, and corresponds to experimental results with threshold current of about $45 \mathrm{~mA}$, due to the large cavity and coupling losses. Results of operation as a transmitter is performed with direct modulation via the injection current at $2.5 \mathrm{Gbit} / \mathrm{s}$. The simulated eye diagram after 100 round trips is displayed in Fig. 6(b) after $2.5 \mathrm{GHz}$ low pass filtering and compared with measurements (Fig. 6(c)), showing agreement and clear eye opening due to good recirculating modulation cancellation.

This first qualitative analysis of the self-tuning transmitter has been followed by quantitative experiments mainly represented by BER measurements, which are detailed in the next section.

\section{Network-embedded self-tuning transmitter performance}

The proposed two-FR architecture has been tested exploiting Gaussian AWGs with 50 to $200 \mathrm{GHz}$ FWHMs and a specifically designed MQW RSOA with small signal gain of $30 \mathrm{~dB}$ (at $100 \mathrm{~mA}$ bias), more than 20-dB PDG and 4-GHz E/O bandwidth.

For $1.25 \mathrm{Gbit} / \mathrm{s}$ and $2.5 \mathrm{Gbit} / \mathrm{s}$, the impact of cavity output coupling on the Optical Budget (OB) has been evaluated [22]. Choosing an output coupling ratio of 50/ $50 \mathrm{OB}$ was measured at $10^{-3}$ BER with propagation up to 100-km SSMF and AWG FWHM of $100 \mathrm{GHz}$, Table 2.

At $5 \mathrm{Gbit} / \mathrm{s}$ the BER curves, for a 420-m drop fibre, are displayed in Fig. 7. Propagation is limited to $10 \mathrm{~km}$ of SSMF 
a)
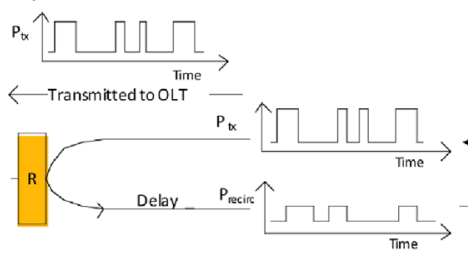

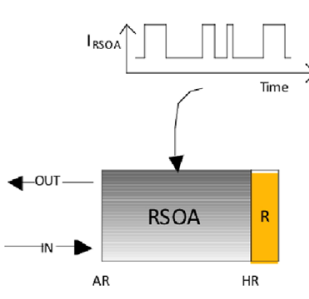

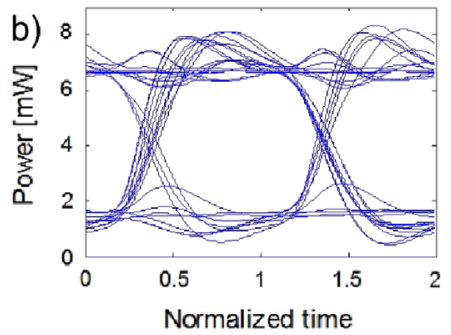

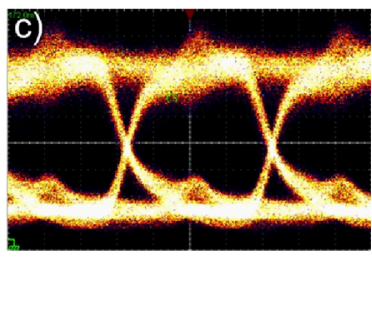

Fig. 6. (a) Schematic of self-seeded cavity. (b) $2.5 \mathrm{Gbit} / \mathrm{s}$ simulated eyediagram ( $I_{\text {bias }}: 130 \mathrm{~mA} I_{\text {mod }}: \pm 50 \mathrm{~mA}$ ). (c) Output eyediagram of a $2.5 \mathrm{Gbit} / \mathrm{s}$ self-tuning transmiter.

Table 2

$\mathrm{OB}$ in $[\mathrm{dB}]$ measurements at $10^{-3} \mathrm{BER}$.

\begin{tabular}{lllllll}
\hline & Cavity length & BTB & $25 \mathrm{~km}$ & $50 \mathrm{~km}$ & $75 \mathrm{~km}$ & $100 \mathrm{~km}$ \\
\hline $1.25 \mathrm{Gbit} / \mathrm{s}$ & $10 \mathrm{~m}$ & 35 & 35 & 34.8 & 34.5 & 34 \\
& $1 \mathrm{~km}$ & 33.3 & 33.1 & 33 & 32.9 & 32.8 \\
& $5 \mathrm{~km}$ & 30.8 & 30.2 & 30 & 29 & 27.8 \\
$2.5 \mathrm{Gbit} / \mathrm{s}$ & $10 \mathrm{~m}$ & 34 & 33.3 & 32.6 & 27.2 & - \\
& $1 \mathrm{~km}$ & 32.4 & 32 & 29.5 & - & - \\
& $5 \mathrm{~km}$ & 30 & 28 & - & - & - \\
\hline
\end{tabular}

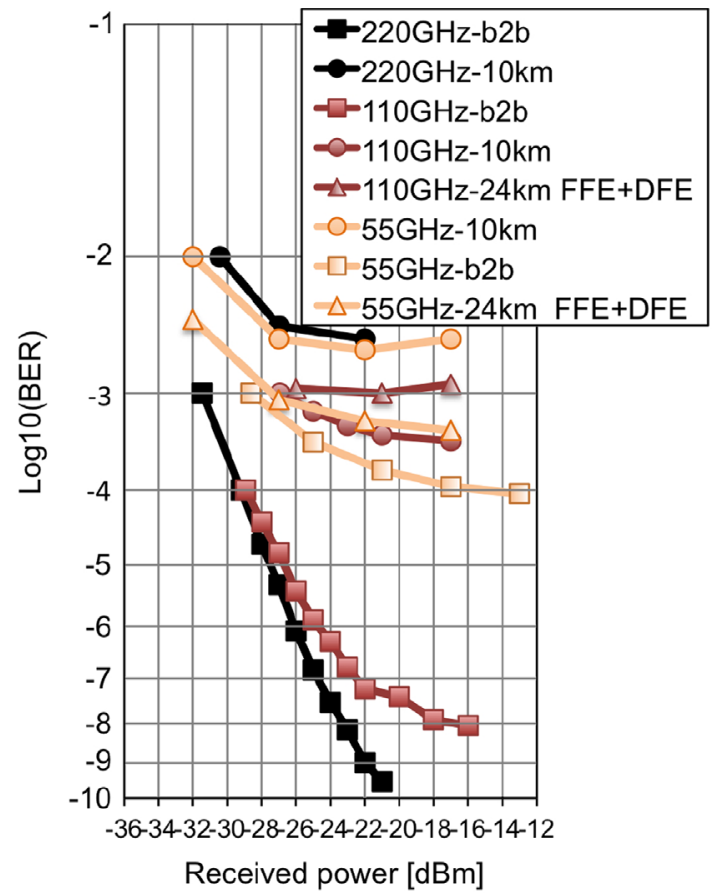

Fig. 7. BER measurements at $5 \mathrm{Gbit} / \mathrm{s}$.

without any electronic digital equalisation. In back-to-back for BER up to $10^{-6}$ the two largest AWG FWHMs have similar performance. With $55 \mathrm{GHz}$ filtering the error floor rises to $10^{-4}$. After propagation, the chromatic dispersion penalties favour narrower bandwidth AWGs. After 10-km SSMF the best trade-off between chromatic dispersion and RIN penalties is obtained for the $110-\mathrm{GHz}$ AWG. Nevertheless using digital electronic equalisation, namely a 9-taps feed forward equalisation (FFE) and a 4-taps

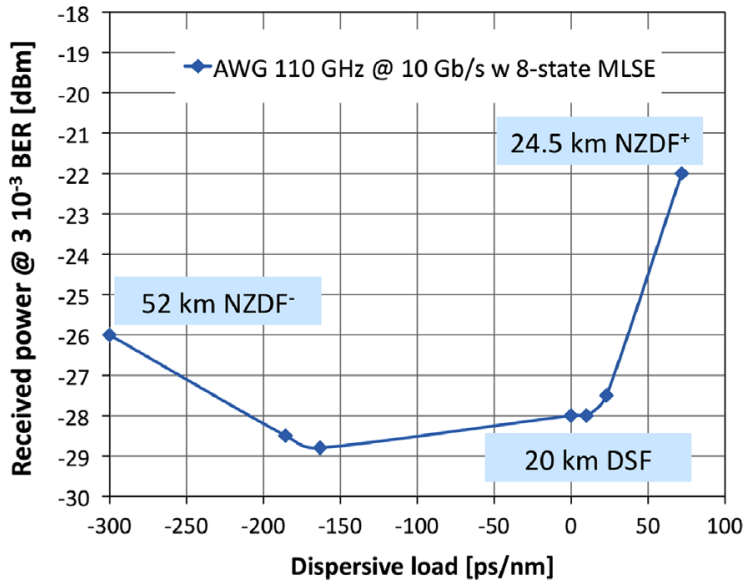

Fig. 8. Received power to achieve FEC limit for different dispersive loads, exploiting MLSE equalisation at $10.7 \mathrm{~Gb} / \mathrm{s}$.

decision feedback equalisation (DFE), 24-km transmission can be bridged.

Finally we evaluated the BER performance at $10.7 \mathrm{Gbit} / \mathrm{s}$ at $3 \times 10^{-3}$ BER of a $110-\mathrm{GHz}$ FWHM AWG cavity transmitter with positive and negative dispersive loads exploiting digital electronic equalisation performed by an 8-state maximum likelihood sequence estimator (MLSE). The results are displayed in Fig. 8: the transmitter with 110GHz AWG allows to bridge up to $75-\mathrm{ps} / \mathrm{nm}$ positive dispersion load and up to $300 \mathrm{ps} / \mathrm{nm}$ negative dispersion load, which have been experimentally obtained respectively with $24.5-\mathrm{km} \mathrm{NZD}^{+}$and $52-\mathrm{km} \mathrm{NZD}^{- \text {feeder }}$ fibres. The $8-\mathrm{ps} / \mathrm{nm}$ experimental point is otherwise obtained by $20-\mathrm{km}$ DSF, which highlights an almost negligible penalty with respect to the back to back. The asymmetric behaviour as a function of the sign of the dispersive load enlightens, on the other hand, that the main limitation to propagation in this case is determined by the chirp associated to the transmitter signal, due to the negative chirp associated with RSOA direct modulation [23].

\section{Conclusion}

Due to the evolution of the radio technologies a new fronthaul segment is appearing between RRH and BBU to achieve a C-RAN architecture. High capacity, low latency, and specific values for jitter and synchronisation are required for the fronthaul based on an optical fibre distribution network, 
which connects the antenna site to the BBU hotel. This optical network has to be optimised in order to make an efficient usage of fibres and minimise deployment cost. Moreover to deal with the high number of digital radio over fibre links per antenna site (one potential link per carrier, per radio sector, per mobile generation), this optical network requires the exploitation of a multiplexing technique: the WDM technology represents the most promising multiplexing solution due to other technologies limitations in terms of power supply and ultimate data rate. We thus proposed a WDM solution based on self-seeded RSOAs, providing compact, colourless and self-tuning transceivers, which will favour the decrease of inventory, operation, administration, and maintenance costs.

We described the development of the network embedded self-tuning colourless transmitter by illustrating the architecture principle of operation, which allows the exploitation of high performance RSOAs having HPDG, together with the actual development of the active chips. We have finally shown experimental performance measurements of the proposed transmitter, which exceed previously demonstrated data rates [24], evidencing capability up to $10 \mathrm{Gbit} / \mathrm{s}$. These results are encouraging for the exploitation of the WDM multiplexing technology in the fronthaul.

\section{Acknowledgements}

The research leading to these results has received funding from the EU FP7/2007-2013 fund under Grant agreement ERMES no. 2885422012.

\section{References}

[1] A. Lometti, G. Cazzaniga, S. Frigerio, L. Ronchetti, Synchronization techniques in backhauling networks, in: Proceedings of the 15th International Transparent Optical Networks (ICTON), 2013.

[2] A. Lometti, V. Sestito, Optical access architectures for backhauling of broadband mobile networks, in: Proceedings of the XVth International Telecommunications Network Strategy and Planning Symposium (NETWORKS), 2012

[3] A. Lometti, C. Colombo, S. Frigerio, V. Sestito, Network architectures for CPRI backhauling, in: Proceedings of the 14th International Conference on Transparent Optical Networks (ICTON), 2012.

[4] C.-H. Lee, W.V. Sorin, B.Y. Kim, Fibre to the home using a PON infrastructure J. Lightwave Technol. 24, 4568-4583, ( 2006).

[5] K. Grobe, J.-P. Elbers, PON in adolescence: from TDMA to WDM-PON, IEEE Commun. Mag. 46 (2008) 26-34.
[6] C. Lee, S. Lee, K. Choi, J. Moon, S. Mun, K. Jeong, J. Kim, B. Kim, WDMPON experiences in Korea [invited], J. Opt. Netw. 6 (2007) 451-464.

[7] J.-I. Kani, Enabling technologies for future scalable and flexible WDM-PON and WDM/TDM-PON systems, IEEE J. Sel. Top. Quantum Electron. 16 (2010) 1290-1297.

[8] Ki-Man Jung-Hyung Moon, Sil-Gu Choi, Mun, Chang-Hee Lee, An automatic wavelength control method of a tuneable laser for a WDM-PON, IEEE Photonics Technol. Lett. 21 (2009) 325-327.

[9] G. Talli, D. Cotter, P.D. Townsend, Rayleigh backscattering impair-ments in access networks with centralised light source, Electron. Lett. 42 (2006) 877-878.

[10] K. Akimoto, J. Kani, M. Teshima, K. Iwatsuki, Super-dense WDM transmission of spectrum-sliced incoherent light for wide-area access network, J. Lightwave Technol. 21 (2003) 2715-2722.

[11] E. Wong, et al., Directly modulated self-seeding reflective semiconductor optical amplifiers as colourless transmitters in WDM PON, J. Lightwave Technol. 25 (2007) 67-74.

[12] A. Pizzinat, P. Chanclou, F. Le Clech, B. Landousies, FTTx where x stays for Antenna: requirements on optical access/distribution network for new mobile backhaul architectures, in: Proceedings of the FTTx SUMMIT Europe, London, UK, 25th April 2012.

[13] A. Pizzinat, P. Chanclou, F. Frank, B. Charbonnier, P. Niger, B. Land-ousies, P. Herbelin, J.M. Picard, J.-P. Charles, Infrastructure conver-gence for fixed and mobile access networks, in: Proceedings of the OFC 2009, Workshop Migration Scenarios toward Future Access Networks I, San Diego, 22nd March 2009.

[14] Common Public Radio Interface (CPRI), Interface Specification, v5.0. Available at: 〈http://www.cpri.info $\rangle, 2011$.

[15] 〈http://www.obsai.com〉.

[16] China Mobile Research Institute, C-RAN The Road Towards Green RAN White Paper Version 2.5. 〈http://labs.chinamobile.com/cran/ wp-content/uploads/CRAN_white_paper_v2_5_EN\%281\%29.pdf), October 2011

[17] P. Chanclou, et al., Optical fibre solution for mobile fronthaul to achieve cloud radio access network, in: Proceedings of the Future Network and Mobile Summit 2013, Lisbon, Portugal, 3-5 July 2013.

[18] L. Marazzi, et al., Network-embedded self-tuning cavity for WDMPON transmitter, Opt. Express 20 (2012) 3781-3786.

[19] M. Presi, E. Ciaramella, Stable Self-seeding of R-SOAs for WDMPONs, in: Proceeding of the OFC 2011, paper OMP4.

[20] M. Martinelli, L. Marazzi, P. Parolari, M. Brunero, G. Gavioli, Polarization in retracing circuits for WDM-PON, IEEE Photonics Technol. Lett. 24 (2012) 1191-1193.

[21] S.Ó. Dúill, L. Marazzi, P. Parolari, R. Brenot, C. Koos, W. Freude, J. Leuthold, Efficient modulation cancellation using reflective SOAs, Opt. Express 20 (2012) 587-594.

[22] Q. Deniel, et al., Self-seeded RSOA based WDM-PON transmission capacities. in: Proceedings of the OFC/NFOEC, Anaheim, CA, 2013, Paper OW4D.3.

[23] L. Marazzi, P. Parolari, M. Brunero, A. Gatto, M. Martinelli, R. Brenot, S. Barbet, P. Galli, G. Gavioli, Up to $10.7-G b / s$ High-PDG RSOA-based colourless transmitter for WDM networks, IEEE Photonics Technol. Lett. 25 (2013) 637-640.

[24] F. Xiong, W.-D. Zhong, M. Zhu, H. Kim, Z. Xu, D. Liu, Characterization of directly modulated self-seeded reflective semiconductor optical amplifiers utilized as colourless transmitters in WDM-PONs, J. Lightwave Technol. 31 (2013) 1727-1733. 